\title{
Erratum to "An ADRC Method for Noncascaded Integral Systems Based on Algebraic Substitution Method and Its Structure"
}

\author{
Zhijian Huang $\mathbb{D}^{1,2}$ Yudong Li, ${ }^{1}$ Yihua Liu, ${ }^{1}$ Bowen Sui $\mathbb{D}^{1},{ }^{1}$ and Guichen Zhang $^{1}$ \\ ${ }^{1}$ Lab of Intelligent Control and Computation, Shanghai Maritime University, Shanghai 201306, China \\ ${ }^{2}$ Department of Electrical, Computer, and Biomedical Engineering, University of Rhode Island, Kingston, RI 02881, USA \\ Correspondence should be addressed to Zhijian Huang; zjhuang@shmtu.edu.cn \\ Received 4 July 2019; Accepted 7 July 2019; Published 15 July 2019 \\ Copyright (C) 2019 Zhijian Huang et al. This is an open access article distributed under the Creative Commons Attribution License, \\ which permits unrestricted use, distribution, and reproduction in any medium, provided the original work is properly cited.
}

In the article titled "An ADRC Method for Noncascaded Integral Systems Based on Algebraic Substitution Method and Its Structure" [1], there was an error in the name format of the fourth author as it should be "Bowen Sui" instead of "Wenbo Sui". The corrected name format is shown above.

\section{References}

[1] Z. Huang, Y. Li, Y. Liu, W. Sui, and G. Zhang, "An ADRC method for noncascaded integral systems based on algebraic substitution method and its structure," Mathematical Problems in Engineering, vol. 2018, Article ID 3905879, 10 pages, 2018. 


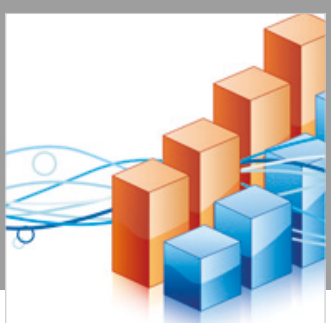

Advances in

Operations Research

\section{-n-m}
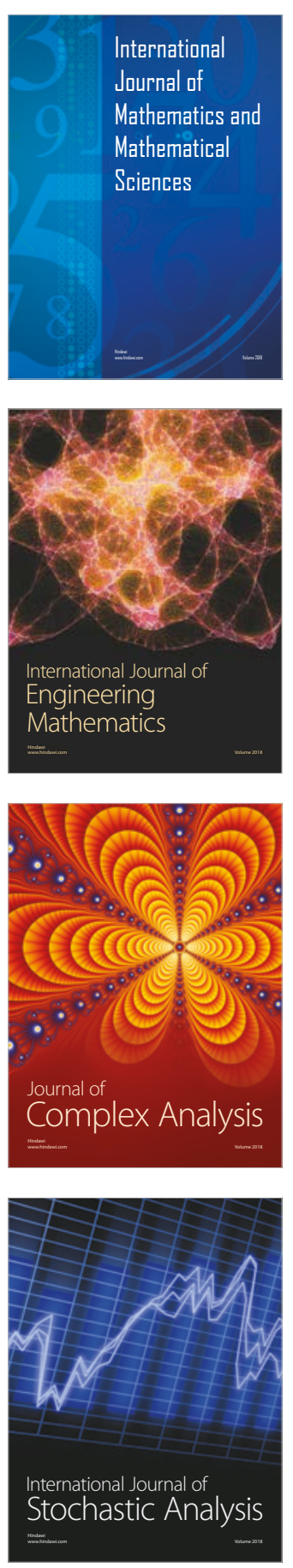
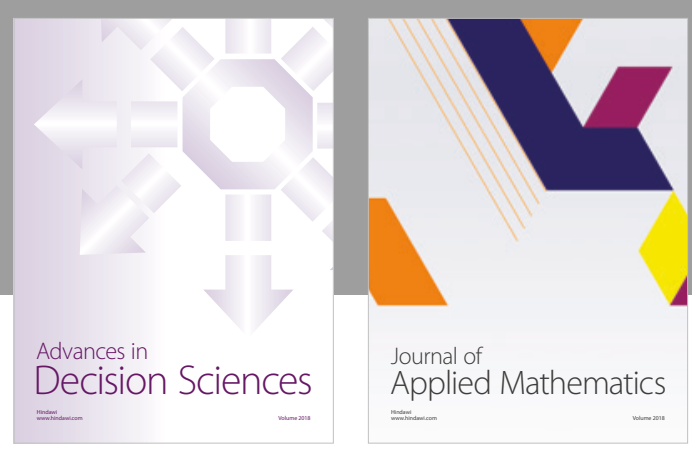

Journal of

Applied Mathematics
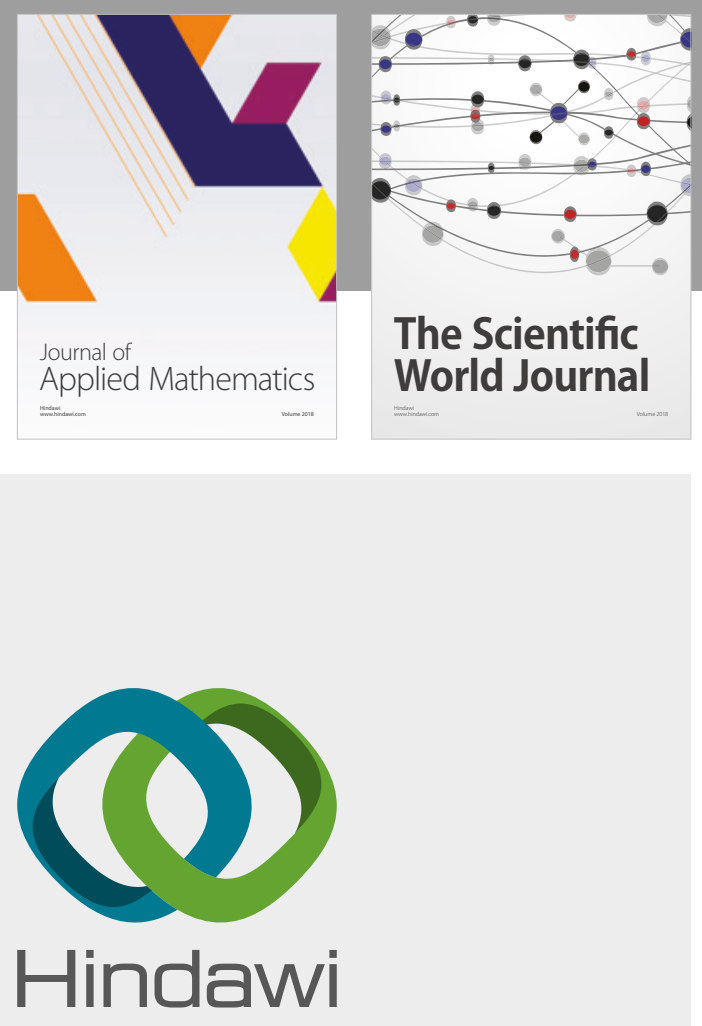

Submit your manuscripts at

www.hindawi.com



Advances in
Numerical Analysis
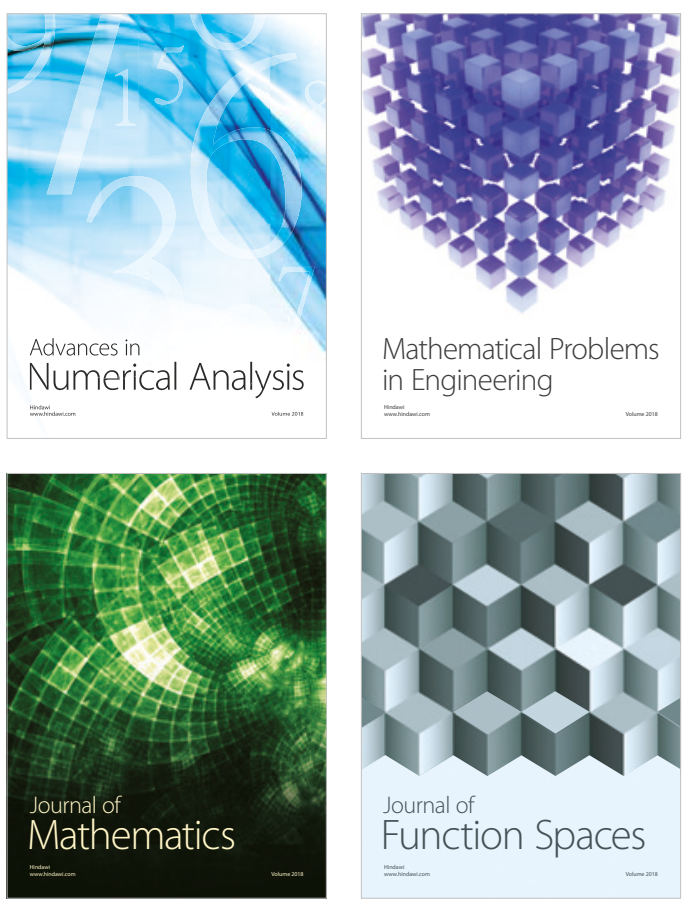

Mathematical Problems in Engineering

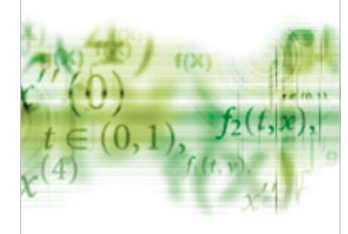

International Journal of

Differential Equations

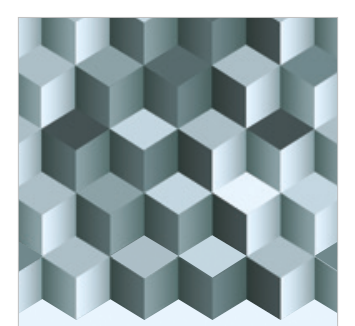

Journal of

Function Spaces

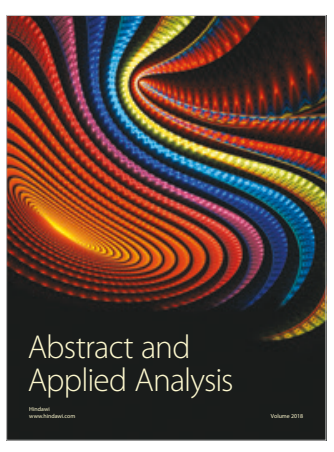

The Scientific

World Journal

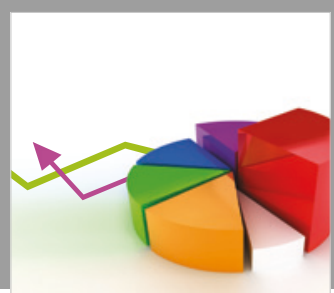

Journal of

Probability and Statistics
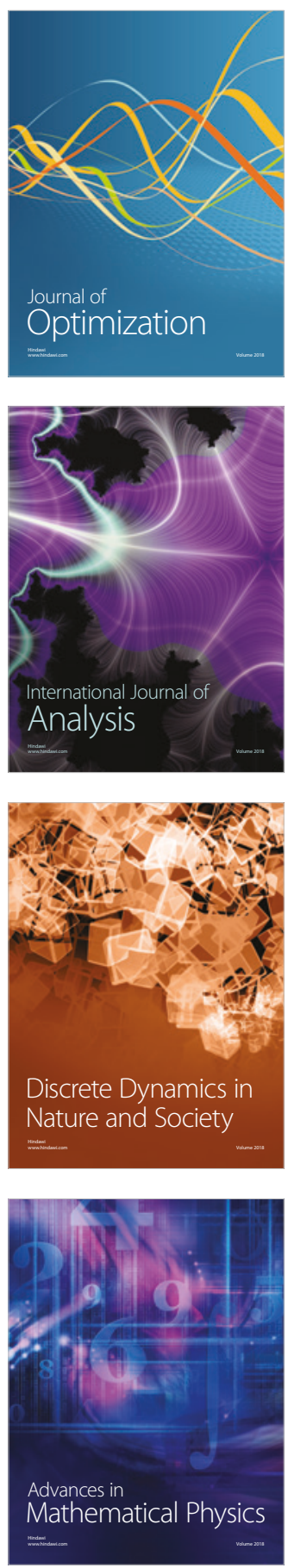